\title{
A Privacy-Preserving Message Forwarding Framework with key caching for low latency
}

\author{
Prasanthi G, G Srinivasa Rao
}

\begin{abstract}
For exchanging messages over opportunistic exchanges in cloud calculating-empowered Internet of Things (IoT), opportunistic Cloud of Things (CoT) is encouraging for customers by means of an emergent conveying policy. In recent times, for predicting upcoming interactions by the determination of enlightening message promoting effectiveness as well as system quantity, several informally-awake structures have been placed onward, influencing consumers' communal features as well as interaction account. Nevertheless, in the extrapolation procedure as well as communication phase of unprincipled CoT distinct secrecy remains commonly ignored. Towards assuring distinct secrecy as well as improving communication effectiveness, in this broadsheet, we develop a secrecy preservative communication promoting context aimed at unprincipled CoT. For improving transmission effectiveness of incurable customers, we mainly assemble twofold-level design of a cloud server. The proposed method can efficiently safeguard distinct secrecy through incorporating a safety-centered flexibility extrapolation procedure using an overpowering assessment procedure. This paper also introduces data key caching to reduce the latency during the transmission process. The proposed method outperforms the conventional methods.
\end{abstract}

Keywords: Cloud of things, individual privacy, mobility prediction, message forwarding, opportunistic networking, key caching.

\section{INTRODUCTION}

By means of a present transmission policy, Internet of Things (IoT) stands favorable for bringing persistent Internet admittance aimed at people and recognize insolent towns [1], [2]. Towards enabling transmissions in IoT, unprincipled interacting remains a model of expertise. For sharing data as well as information [3], personalities in immediacy can remain associated through their undersized-choice policies. Towards enabling transmissions using unprincipled interacting in cloud calculating-empowered IoT, we apply Opportunistic Cloud of Things (CoT). Accordingly, aimed at cellular systems, unprincipled CoT has established by means of a new design to improve loads. In additional arguments, unprincipled CoT remains suitable aimed at recurrent comparability, wherever mobile nodules (e.g. consumers carrying smart phones)

Revised Manuscript Received on October 30, 2019.

* Correspondence Author

Prasanthi G*, Research Scholar, Department of Information Technology, GITAM University, Visakhapatnam (A.P), India. Email: prasanthi.g946@gmail.com

G Srinivasa Rao, Assoc. Professor, Department of Information Technology, GITAM University, Visakhapatnam (A.P), India. Email: giduturisrinivasarao74@gmail.com

(C) The Authors. Published by Blue Eyes Intelligence Engineering and Sciences Publication (BEIESP). This is an open access article under the CC-BY-NC-ND license http://creativecommons.org/licenses/by-nc-nd/4.0/

could interconnect by each other using Bluetooth or else additional wireless transmission expertise resourcefully [4]. In preference to transferring information commencing a website reliably, Nodes can stake substances by means of a "store-carry-and-forward" device. Unprincipled CoT consumes extensive submission projections. For instance, for sharing collective-means fillings amid mobile customers by means of unprincipled associates [5] MIT Media Lab builds a scheme, called CoCam. Vehicular unprincipled systems must remain advanced [6] using the severe increasing of intellectual carriages. Towards supporting unprincipled transmissions amongst automobiles, the disposition of Intelligent Transportation Systems (ITS) has remained stimulated in Japan. Japanese government has organized beyond 1000 Road Side Units (RSUs) mostly about a public road [7]. Numerous kinds of fillings, such as existent-period broadcast, climate reportage, as well as movies can remain distributed in unprincipled CoT. Especially, a server provider may prefer to deliver contents to a specified fraction of mobile users. These contented owners at that time travel round for exchanging fillings using additional mobile consumers resourcefully. Subsequently, effective message promoting appliances remain substantial aimed at unprincipled CoT [8]. At this time, towards concerning about individual discretion it remains unprecedentedly usual aimed at entities. For participating in message promoting procedures of unprincipled CoT, confidentiality-preservative devices remain significant conditions aimed at operators. Nevertheless, planning an effective as well as secrecy-preservative message promoting method faces numerous encounters:

1) By what means to aptly as well as efficiently onward messages amongst vehicles as well as additional individuals using undersized-expanse wireless transmission expertise remains stimulating. Existing investigates usually adopt that vehicles can remain associated towards dissimilar individuals in an appropriate method. Conversely, owing towards the unprincipled transmission design [9], they might continuously stay separated using these individuals aimed at an extended period. This might affect in outbreaks certainly propelled using mischievous nodules. Hence, a new structure desires to remain assembled.

2) How to defend the isolated data included in a relation forecast procedure remains stimulating. To overthrow difficulties conveyed using the energetic environment of unprincipled CoT, such as extreme distribution invisibility, small distribution proportion as well as reserve ingesting, link extrapolation procedures remain usually employed. 


\section{A Privacy-Preserving Message Forwarding Framework with key caching for low latency Cloud of Things}

Nevertheless, by the side of mutual interaction stage (comprising interaction period as well as frequency) and social-stage

(comprising node approval as well as significance), a connection calculation procedure might include certain distinct data. Assailants might simply ill use such isolated data if obstruction strategies doesn't remain in position. As system safety as well as effectiveness tends to resist with each other, yet, confidentiality as well as safety concerns in a relation (or else flexibility) forecast procedure have attained slight consideration regarding unprincipled CoT to this point.

3) Grounded on an extremely-protected as well as simply-employed cryptosystem, a major involvement should remain absorbed on by what means to retain information secrecy. Several investigates have concentrated on either fictitious name-grounded expertise or else cryptographic approaches. Although k-anonymization is usually practiced in a fictitious name structure [10], Homomorphism encoding procedures as well as elliptic curvature encoding expertise remains dual effective implements in a cryptographic structure. However, various patterns involve compound system scheming, in addition others claim fairly a firm system atmosphere. Let's say, to finish a fictitious name modernizing procedure, certain fictitious name-grounded pattern involves that certain entities exist in a region, if not the assailants may still locate an operator. In message promoting aimed at opportunistic CoT, these circumstances might grounds small distribution proportion as well as extreme distribution interval.

4) For pursuing excess yield, self-centered as well as mischievous nodes might fake or even alter their attained recompenses. By what means to assure the safety of consumers' recompenses be worthy to be deliberated, such that self-centered as well as mischievous operators do not disturb the objectivity of enticement systems. Towards guaranteeing both message distribution effectiveness as well as distinct secrecy, we lay onward a Privacy-preserving message promoting framework aimed at Opportunistic cloud of things (PCON) for addressing the tasks stated overhead. Both customary information secrecy as well as characteristic-grounded secrecy (i.e., a consumer's interaction-stage and communal-stage data) is deliberated aimed at opportunistic CoT. The divisional load engaged upon a wireless customer can be mostly discharged through our double-level cloud server. Simultaneously, the basic managing as well as authentication can be accomplished virtually in an appropriate method in an interrelated wireless atmosphere. Towards choosing the succeeding-hop transmit node, using the resolution of safeguarding communal characteristics convoluted in the transmission relations amongst nodes, we incorporate a safety-grounded flexibility forecast procedure using the routing resolution procedure.

\section{RELATED WORK}

We mainly analyze certain demonstrative mobility forecast procedures aimed at communication effectiveness development so as to accomplish opportunistic communication of CoT. At that time, to encourage entities in CoT aimed at supportive message promoting, we demonstrate present inducement information promoting

structures. Through the determination of confirming safety for CoT communication, we accomplish the discussion using certain prevailing secrecy preservative approaches.

\section{A. Mobility Prediction Algorithms}

Aimed at opportunistic CoT, the indeterminate relation configuration and mobility of nodes points to blind and incompetent message promoting deeds [13]. To enhance communication effectiveness, determinations have been prepared on relation forecast concerns. For improving opportunistic message promoting capabilities in vehicular systems, ZOOM scales the flexibility of vehicles [14]. Consistent with communal-stage and interaction-stage mobilities, mobility forecast implementation is considered in the direction of onward messages. For providing a standard for message promoting comprising periodical interaction period as well as spatial-chronological delivery utilities of connections, Resolutions grounded on interaction-stage data anticipate to forecast information aimed at node combines. Approaches grounded on communal stage data focus on a system configuration towards making messages carried to nodes using an extraordinary possibility to come across others. To forecast come across period, interaction frequency as well as non-periodic interacts in [15]. The aforementioned can both enhance the distribution proportion as well as reduce the routing price, Periodic design excavating pattern as well as assessment trees remain employed. A flexibility-alert geocast method, called GeoMob [16], proposes towards handling great flexibility as well as temporary comparability in vehicular systems. Producing GeoMob accessible and transmission-operative, the writers incorporate vehicle flexibility data into dissimilar stages. Nevertheless, safety as well as confidentiality matters have not stood considered into description in entirely the flexibility-forecast-grounded procedures. As distinct confidentiality might be exposed to mischievous nodes, in fact it remains stimulating to accomplish those approaches in actuality.

\section{B. Incentive Data Forwarding Schemes}

Through the determination of redeeming their restricted system possessions, Self-centered nodes remain abundant in opportunistic CoT that remains unwilling to onward messages. The repute upsurges once a node onwards messages effectively in repute-centered enticement methods. SUCCESS targets towards stimulating self-centered nodes to transmit messages honestly by means of a repute-centered enticement procedure [17]. To stimulate nodes towards transfer messages, simulated cash remains usually influenced in acknowledgement-grounded structures. Towards encouraging consumers to contribute in message distribution in Delay Tolerant Networks (DTNs), in addition to a simulated form is presented for acclaims [18], a self-interest-driven incentive scheme is proposed. In Tit-For-Tat schemes, encountered nodes are essential to interchange the similar no. of messages or weights. For instance, for promoting the content distribution procedure in DTNs [19], Mobi Trade builds an efficacy-determined trade structure. Additionally, certain communal-grounded message promoting structures have remained anticipated, 
considering the compensations of communal relations for improving message distribution effectiveness.

For instance, a communal-alert opportunistic routing in mobile communal systems, called CAOR, comprises a home-alert exemplary and categorizes nodes conferring towards their communal assemblies in opportunistic CoT [20]. To kindle self-centered nodes who might onward messages grounded on system groups CAIS assumes simulated acclaim [11]. A dual-stage routing procedure grounded on an attention-compelled flexibility exemplary has remained anticipated in [21], where human performance configurations and position inclination remain deliberated. The aforementioned resolutions are completely grounded on dissimilar illustrations of communal implication, that is, nodes are essential to deliberate communal data (e.g., battery level, profession and attention) towards making choices on information promoting. Inappropriately, these communal characteristics remain susceptible as well as informal to be mistreated by mischievous nodes.

\section{Privacy Preserving Methods}

In what manner we can assure distinct confidentiality appeals great consideration in current ages. Presently, pointing at avoiding mischievous performances and defending distinct confidentiality-preservative structures have been extensively explored [22]. Usually, distinct confidentiality comprises 3 characteristics for opportunistic CoT, they are, position, information and individuality. Position secrecy depends on consumers' position. Even though individuality one attentions on consumers' contour as well as inclinations, information secrecy remains related to message substances. In view of distinct secrecy, several investigations have concentrated on secrecy-preservative methods. Smart Mask, a background-built structure layer secrecy safeguarding answer is anticipated to inevitably study consumers' secrecy inclinations below dissimilar backgrounds as well as deliver an apparent secrecy device for consumers [23]. The conformation of a secrecy stage remains attained using programmed position outline managing, confined background arrangement and consumer inclination enquiring. To defend consumers' secrecy, an applied collection identical method without engaging any Trust Third Authority (TTA) is anticipated [24]. To lessen the calculation as well as transmission above, an uncertain matrix procedure remains employed to produce consumers' ability rather than cryptographic calculation. However, the safety cannot be completely assured lacking TTA. A latest review of safety as well as confidentiality in the IoT world has been completely deliberated in [25]. The aforementioned attentions on the existing safety and confidentiality concerns met by the IoT structure exemplary, where assailants might influence conceivable structure faults to initiate the outbreaks. To confirm the structure safety, outbreak confrontation outlooks and conviction organization are usually considered as twofold positive answers.

This revision is an addition of our preceding effort in [26]. Paralleled by our preceding effort, we mark the succeeding enhancements: 1) numerous stimulating outbreaks remain deliberated, e.g., information delayed outbreaks. Local Clouds (LCs) are considered as semi-conviction, and might conspire using mischievous nodes; 2) A twofold-layer cloud server remains assembled, and to progress transmission

effectiveness as well as discharge calculation loads for mobile terminals, a complete proposal is delivered; 3) A great productivity procedure aimed at characteristic assessment is considered in a safe flexibility forecast procedure for a routing assessment; 4) By accessing safety strategies for consumers and defending consumers' private information on LCs, An attribute-based cryptographic system is designed; 5) Two datasets are employed to calculate presentations of PCON, CAIS, TRSS as well as Prophet routing. Metrics comprise average distribution proportion, average distribution interval, average distribution price, acclaim assessment and effective distribution proportion.

\section{SYSTEM AND ATTACK MODELS}

Inthisunit, we define the structure as well as outbreak simulations in depth. Fig. 1 demonstrates the structure exemplary of our PCON system. There exist $\mathrm{N}$ mobile nodes, interacting with all other by means of Bluetooth or added transmission frequency resourcefully. To attain their early acclaims, customers are requisite to record to a TTA afore linking with the system. Communal features, such as name, age, profession and interests, remain essential data aimed at registering.

\section{A. Cloud Server Model}

Since the aforementioned consumes a substantial influence on the presentation of message distribution proportion, in what way to assure effective transmission among nodes as well as TTAs in opportunistic CoT has not been responded up till now to our finest information,. In simply dependent on a transmit-and-onward transmission design for opportunistic CoT, it remains slightly stimulating for customers for making appropriate transmissions by additional individuals. We build a dual- stage cloud server exemplary as demonstrated in Fig. 1 , to resolve this difficult. Remind that, for solving the difficult of trustworthy calculating as well as managing, the cloud has remained extensively attended by means of a designation of TTA [27]. The inspected dual-stage Cloud Server (CS) comprises twofold portions: the upper stage is Remote Cloud (RC) and the lowermost one is Local Clouds (LCs). Aimed at easiness, we assume that there exists only one RC, as well as a lengthy expanse survives amongst RC and customers. RC is unswervingly accomplished as well as organized through TTA. The benefits are calculation as well as storing capabilities could be enhanced paralleled by the customary TTA (e.g., [28] and [29]), and the safety and secrecy of customers' data can remain assured. RC drives disconnected once concluding registering. LCs remains influenced for reducing the calculation burden for terminals and enhance transmission effectiveness. We adopt that LCs continuously remains connected. LCs could be positioned in the regions where persons are suitable for visiting, such as coffee bar, school and shopping mall. Wireless transmissions expertise remains influenced towards supporting the transmissions amid LCs as well as customers. Nevertheless, LCs couldn't openly interrelate with nodes exterior to their transmission extent, for example, the two nodes at the bottommost of Fig. 1. Moreover, nodes could continuously interact with each other in their transmission extent. 
The collection of LCs is represented by $\mathrm{C}=\{\mathrm{c} 1, \mathrm{c} 2, \ldots \ldots, \mathrm{cq}\}$ as well as RC using $\mathrm{R}$.

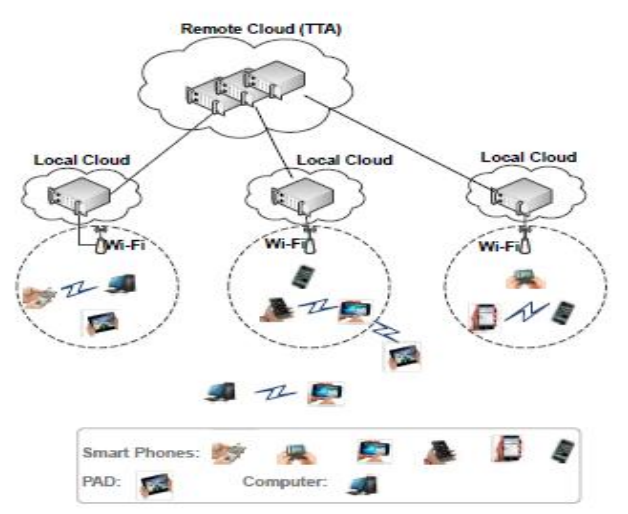

Fig. 1: System model of PCON.

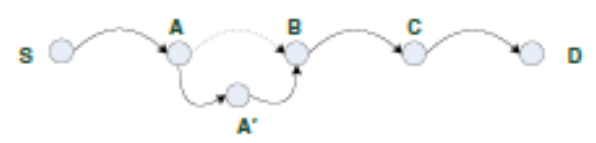

(a) Sybil Attack

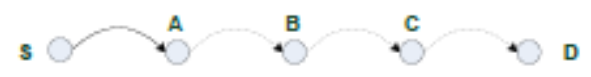

(b) Drop for profit

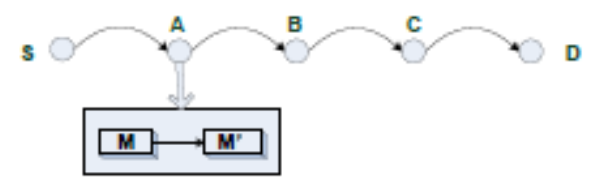

(c) Data Tampered Attack

Fig. 2: Three kinds of attacks.

\section{B. Attack Model}

Three types of nodes exist in the system: Benevolent, self-centered as well as mischievous nodes. Benevolent nodes remain fully supportive in message promoting, whereas self-centered nodes desire to supply messages for customers with a close by affiliation, such as friends, families as well as colleagues. In additional terms, whether their individual or else communal efficacies can be exploited is a basic issue for message transmitting. Unlike from self-centered nodes, mischievous nodes perform unusual deeds ensuing in system condition. As demonstrated in Fig. 2, three types of outbreaks are deliberated as follows:

1) Sybil attack: Towards gaining additional benefit, a mischievous node might copy certain effective nodes and supplement them into a message promoting route. As presented in Fig. 2(a), node A copies a node (named $\mathrm{A}^{\prime}$ ) and places node $\mathrm{A}^{\prime}$ among the aforementioned as well as node $\mathrm{B}$. The compensation gained through node $\mathrm{A}^{\prime}$ remains completely possessed by node A.

2) Drop for profit: An assailant playacts to remain prepared for transmitting messages for extra nodes with the intention of gaining great yield. The message could be noiselessly let go when it is given a message. Fig. 2(b) demonstrates the outbreak procedure of such a node named A that descents the bundle in its safeguard and does not proceeds to interact with node B.
3) Data tampered attack: Through the determination of confusing target nodes, the message matter could remain interfered using mischievous nodes. Moreover, pointing towards getting additional benefit from CS, the information might similarly remain condemned through mischievous nodes. As revealed in Fig. 2(c), assailant A alters message M to $\mathrm{M}^{\prime}$, and later provides message $\mathrm{M}$ ' to additional transmit nodes. Lastly, message $\mathrm{M}^{\prime}$ is conveyed towards the target node. Information, feature, as well as deal remain the 3 features of distinct secrecy that we assume. Although information altered outbreak might root attack of information secrecy as well as operation secrecy, the outbreak of fall aimed at benefit might interrupt information secrecy. Since it remains continuously simple to remain propelled after the secrecy is well preserved using nodes, Sybil outbreak remains inconsistent by distinct secrecy. The quality grounded secrecy might remain disturbed if a benevolent node interconnects with a Sybil node grounded on a mobility forecast method additionally. Hence, the considered method ought not merely safeguard distinct secrecy, but likewise oppose Sybil outbreaks. Moreover, we assume that LCs remains truthful but- inquisitive. They might anticipate obtaining furtive data of customers through introducing inactive outbreaks, deprived of transforming fillings or scheming with RC. Nevertheless, an LC might scheme with mischievous nodes for getting the message in the course of a communication procedure, interrupting information secrecy.

\section{A. Overview of PCON}

PCON purposes to assure the communication productivity as well as safeguard distinct secrecy. Mainly, we first launch a structure exemplary, wherein a Dual-Stage Cloud Server remains qualified. At that time, we place onward a message-promoting appliance grounded on the considered structure, comprising three main measures, i.e., Security-based Mobility Prediction, Message Handler as well as Reward Reporting Process. Security-based Mobility Prediction is intended for finding a promising route from the basis node towards target nodes. In the meantime, it similarly safeguards the feature-grounded secrecy for communicating nodes. A feature-grounded cryptographic procedure is considered in Message Manager with the intention of maintaining information secrecy. Finally, we consider Compensation Reportage Procedure proceedings and Reportages recompenses for passing-by nodes in a cryptographic approach towards maintaining the operation secrecy.

For intensely understanding the method for message promoting in PCON, we describe the aforementioned leading procedure along these lines: assume that whenever a node $v_{i}$ come across $v_{j}$, it calculates whether $v_{j}$ remains appropriate for transmitting messages by means of the subsequent-hop communication node. The meeting nodes equate personality betweenness and connection interval approximation [14] grounded on Security Multiparty Computation (SMC) [30] that permits the secrecy of their elaborated standards. While node $v_{i}$ travels into the transmission region of an $\mathrm{LC} c_{l}$, the aforementioned mainly encodes its native messages using a similarity encoding algorithm, 
for instance, Advanced Encryption Standard (AES), and at that time encodes the basic of AES using an feature-grounded procedure by the support of Message Manager.

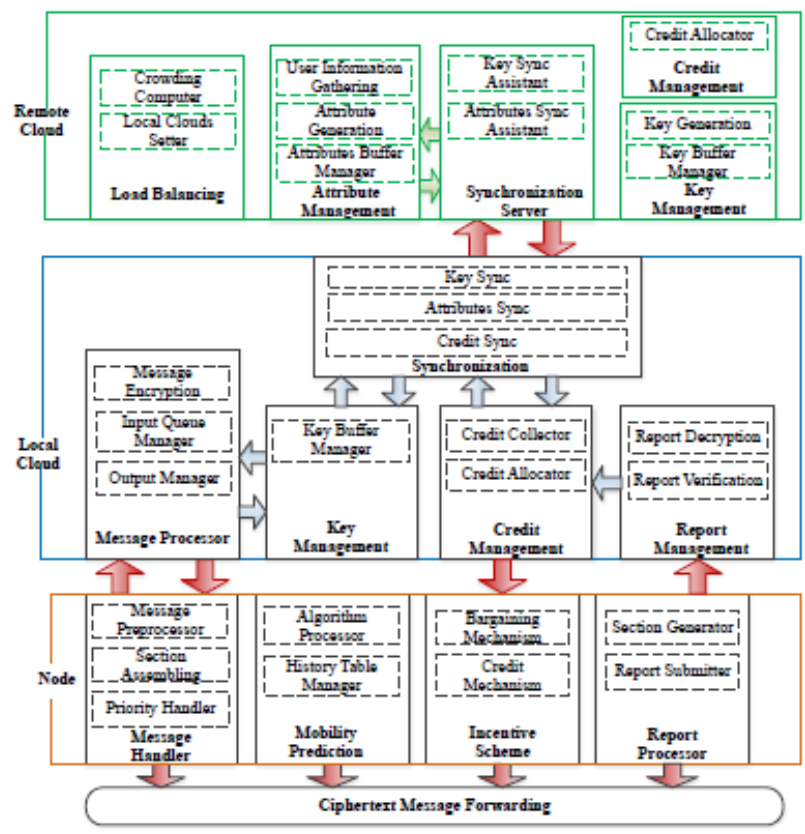

Fig. 3: Structure of PCON.

In the meantime, $c_{l}$ certifies information presented using node $v_{i}$, as well as calculates nodes' recompenses by means of Compensation Reportage Procedure. LC $c_{l}$ similarly re-encodes the bases transferred using nodes, also matches with RC occasionally.

\section{B. Detailed System Design}

We majorly define the modules and then transmission procedure of PCON.

1) System Components:

Aimed at terminal customers, the Cloud computing overlays a novel method for depositing a divisional loads. A mobile scheme can influence a native cloud (also named cloudlet) towards enhancing its ability so as to progress structure presentation. It might remain observed by means of a corresponding technique aimed at the condition that a node remains incapable to openly link with RC. This is for the reason that a wireless connection stage remains impulsive as well as the price for cellular transportation remains costly [31], [32]. We create a dual-stage CS structure, comprising an RC and numerous LCs are stimulated using the overhead statement. The benefits are as follows: 1 ) it could release dense traffic, as divisional loads of customers are discharged towards LCs; 2) the structure remains vigorous. If an LC backfires, others could even ensure the capability to assure the usual system procedure; and 3) it remains inexpensive aimed at entities towards updating their confined statistics by employing small-expanse wireless expertise. As demonstrated in Fig. 3, RC comprises 5 segments, namely, Burden Harmonizing, Feature Managing, Basic Managing, Harmonization Server as well as Acclaim Managing. Burden Harmonizing composures loads amongst LCs through assigning them in suitable positions. Feature Managing maintains controlling of customers' communal features preoccupied from registering data. Basic Managing preserves path of furtive bases in the structure. Harmonization Server aids LCs apprise confined data commencing RC. The entire recognition in the system remains accomplished using Acclaim Managing. An LC comprises 5 modules: Message Processor, Credit Management, Report Processor, Basic Managing as well as Harmonization Segment. The purpose of Message Processor is to encode messages aimed at nodes. Recompenses in the structure are organized using Acclaim Managing. Report Processor authenticates information acknowledged through target nodes, as well as delivers compensation grades to Acclaim Managing. Basic Managing preserves alteration solutions, and offers them towards the additional segments if required. Harmonization Segment informs by means of RC at static intermissions. Four efficient constituents are incorporated in every single node: Message Manager, Flexibility Forecast, Inducement Method as well as Report Processor. Message Manager chooses obtainable messages for communication, and encodes these messages as well as bases fundamentally. Flexibility Forecast moves out a forecast process for guiding message interactions. Enticement Pattern records recompenses in usage of effective currency to inspire nodes towards transferring messages. Report Processor enhances statement units in the last part of messages towards recording recompenses by means of confirmations for authentication.

2) Communications Process:

The transmissions in the created structure may be distributed into 3 groups: (a) transmissions between nodes; (b) transmissions amid a node as well as an LC; (c) harmonization amid an LC as well as RC. Flexibility Forecast attains historic interaction data from a confined safeguard whenever a node formulates to interchange messages by additional one. Significance Manager regulates the promoting command of confined messages if additional node remains appropriate aimed at communication. An Inducement method kindles contestant nodes towards cooperating in information distribution. For recording recompenses of nodes, statement Processor customs a statement unit. At that time, the Unit Gathering component in Message Manager places the statement unit by the end of the message. It mainly verifies whether confined messages remain accessible for communication whenever a node travels into the transmission region of an LC. Otherwise, Message Preprocessor in Message Manager directs entire encoded solutions to Message Processor in the LC over a furtive medium. The furtive medium might remain recognized using prevailing approaches, such as the process in [33]. We ensure not stipulating the equivalent information as it is not our effort in this revision. At that time, Message Processor in LC adjacent recognizes the procedure command in Input Queue Processer, re-encodes solutions using Message Encoding, 
as well as precedes them towards nodes through Productivity Director. A node stocks information in the safeguard, as well as yield to them to an LC through statement manager.

Acclaim Managing assigns acclaims towards nodes once information is authenticated by Statement Administration. Basic Managing follows regulation of conversion bases deposited in the safeguard using Basic Safeguard Administrator.

LCs harmonizes information using RC by means of the Harmonization segment afterwards a customer's registering and maintains a pathway of the recently produced conversion bases. Harmonization Server in RC aids LCs harmonizes. Characteristic Managing transmissions the customer's inputs into features in addition to Basic Administration produce furtive and conversion bases aimed at the customer whenever a customer records to RC. The virtual-encryptions of the modernizing procedure of $\mathrm{RC}$, the provision in an $\mathrm{LC}$ as well as the leading stages of a message promoting procedure aimed at nodes remain demonstrated in Extra Folder.

\section{MESSAGE FORWARDING MECHANISM}

In this unit, we define the message promoting scheme in PCON. Key schemes are briefed in Table1.

TABLE I: Major notations

\begin{tabular}{|c|c|}
\hline Notation & Description \\
\hline$v_{i}$ & Node i \\
\hline$M_{i}$ & $\begin{array}{l}\text { Message collection in buffer } \\
\text { of node i }\end{array}$ \\
\hline$m_{\text {ii }}$ & $\begin{array}{l}\text { The ith message in the } \\
\text { message collection of node i }\end{array}$ \\
\hline $\mathrm{C}$ & Group of LCs in the network \\
\hline$c_{i}$ & The ith LC in the network \\
\hline $\mathrm{R}$ & The remote cloud \\
\hline$M_{\mathrm{i}}^{e}, \bar{M}_{\mathrm{i}}^{\ominus}$ & $\begin{array}{l}\text { Message list in the buffer of } \\
\text { node i }\end{array}$ \\
\hline $\mathrm{S}$ & A user's attribute set \\
\hline$S_{i}$ & $\begin{array}{c}\text { The ith attribute in a user's } \\
\text { attribute set }\end{array}$ \\
\hline$v_{\mathrm{i}}, k_{\mathrm{i}}$ & The value of ith attribute \\
\hline$p_{i}$ & Public key of node i \\
\hline$S_{i}$ & Private key of node i \\
\hline$T_{\mathrm{i}}$ & Transformation key of node i \\
\hline$\gamma_{i}$ & Access tree of node i \\
\hline$\overline{D_{i} d d}$ & $\begin{array}{l}\text { The contact delay estimation } \\
\text { from node I to destination } \\
\text { node } \mathrm{j}\end{array}$ \\
\hline$C_{i}$ & $\begin{array}{l}\text { The ego betweenness of node } \\
\text { i }\end{array}$ \\
\hline$D^{\min }$ & $\begin{array}{l}\text { The shorter value out of two } \\
\text { compared values for contact } \\
\text { delay estimation }\end{array}$ \\
\hline$C^{\min }$ & $\begin{array}{c}\text { The shorter value out of two } \\
\text { compared values for ego } \\
\text { between centrality }\end{array}$ \\
\hline
\end{tabular}

\section{A. Security-based Mobility Prediction}

By the intention of enhancing communication effectiveness, boundless considerations have been drained aimed at flexibility forecast procedures. Information or else message promoting procedures, concentrating on any interaction-stage or communal- stage flexibility of convoluted nodes, have remained completely explored. Nevertheless, existing flexibility-grounded forecast procedures usually disregard the characteristic-grounded secrecy elaborated in a forecast procedure. We place onward a safety-centered flexibility forecast technique in allowing collaboration by suspicious associates with the intention of preserving this type of secrecy. The inspected method is a difficult of Yao's Millionaires [34].

We assume 2 nodes $v_{i}$ and $v_{j}$ in connection, as demonstrated in Fig. 4. Let $M_{i}=\left\{m_{i 1}, m i 2, \ldots, m_{i r}, \ldots m_{i P}\right\}$ represent the messages in node $v_{i}$ 's safeguard. The network layer of node $v_{i}$ remains accountable for defining whether a message in $\mathrm{M}_{\mathrm{i}}$ ought to be promoted to node $\mathrm{v}_{\mathrm{j}}$. Here moreover exists a representative in the submission level, accomplishing the flexibility forecast procedure to forecast the upcoming connection as well as response consequences towards the system level.

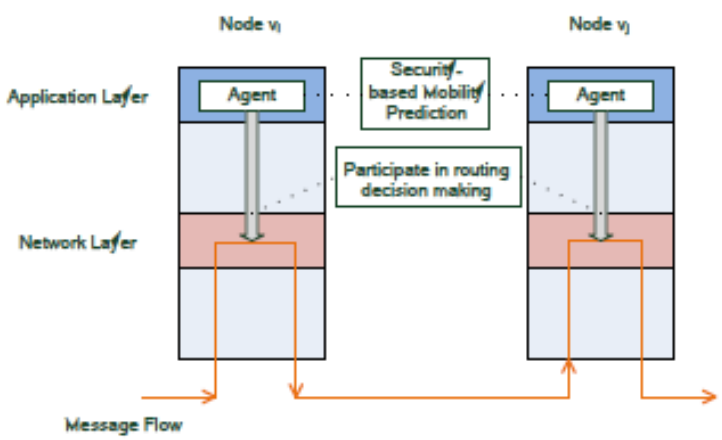

Fig. 4: Message routing.

In incorporating data from both the network as well as application stages, node remains capable for making an appropriate choice aimed at message communication. Grounded on which the succeeding connection can remain projected, we instruct a k-direction Markov series to pretend meeting position aimed at dual nodes rendering towards their previous connections. Whenever nodes as well as come across, twofold fundamentals are paralleled, i.e, connection interval approximation $L(L)$ as well as self-esteem betweenness ( ). A projected significance of connection interval from node towards target node is well-defined by means of connection interval approximation $L$, whereas self-esteem betweenness remains a communal measured illuminating the significance of in a system. If Iremains little, node remains designated by means of the subsequent-hop transmit node, and we create $D^{\text {min }}=I$. If $L$ and $L$ doesn't occur, a relationship amongst as well as is accomplished. The node by a complex significance will remain designated towards carrying the message, and

$$
C^{\min }=\min \left\{C_{i}, C_{j}\right\}
$$




\section{B. Message Handler}

Message Handler progresses messages afore they are referred out. Message Handler majorly encodes confined messages using AES, as well as encodes the basic of AES using a characteristic-grounded procedure locally once a node travels into the wireless transmission extent of an LC.

At that time, it surrenders the encoded basic towards an LC. The encoded basic extents the component of Message Processor on the LC sideways, and is re-encoded by means of a conversion basic. The node directs the encoded messages as well as the re-encoded basic towards the subsequent-hop transmit node composed afterwards unloading the re-encoded basic. Customary cryptographic approaches involve bases to remain modernized occasionally, not only overriding huge system possessions but then again enhancing the divisional complication. We place onward a characteristic-grounded cryptographic procedure that could assure structure safety using dependable bases as well as admission strategies, deprived of the necessity of episodic basic informing. We describe 3 types of bases, i.e., isolated, communal and conversion bases. The benefits of our structure are as trails: a) mainstream of calculation jobs centered on the cryptographic procedure remain traveled to LCs, confirming the least calculation aimed at terminals; b) we describe a conversion basic towards re-encoding bases aimed at a similar encoding procedure, constructing LCs attentive of not any customer's isolated data towards guaranteeing distinct secrecy. Whenever node $v_{i}$ produces a message $m_{i}$, it mainly encodes $m_{i}$ using a regularity encoding procedure by means of key $E_{i}$, e.g., AES. Moreover, it similarly encodes basic $E_{i}$ grounded on the communal basic and characteristics of the target. The complete application is as trails: Mainly, we choose a polynomial $q_{x}$ aimed at every single node $x$ to practice an admission tree $Y_{i}$. Its basis node $R$ is fixed using an indiscriminate assessment $s \in Z q$, and $q_{R}(0)=s$.

\section{Reward Reporting Process}

Towards encouraging nodes aimed at message communication, a recognition-grounded enticement structure could be employed. They gain certain simulated cash if they are fruitful in communicating messages. Our structure remains companionable by multi-varieties of enticement methods, e.g., a game-theoretic enticement method aimed at transmit assortment facilities [35]. A new arrangement by means of an added message unit remains established in this effort so as to safeguard customers' recompenses. It may file each node's simulated cash as well as safeguard the operation secrecy. The message configuration is specified in Fig. 5. Heading as well as content remain twofold simple portions in a message configuration. Whenever the aforementioned fits into a communicating message, a statement remains affixed towards the message extremity, as presented in figure 5. For instance, every transmit node may enhance a unit by means of a substitute-statement for its operation, comprising unit heading as well as unit content. Segment dimension characterizes the dimension of a segment. Unit content holds 4 components, namely, Node Id, Gain, Preview Node Id and Next Node Id. Node Id is the recognition of the existing transmit node, whereas Gain is the attained recompense of the present transmit node. Preview Node Id is the recognition of an adjacent node, distributing the message to the existing transmit node. Next Node Id is the recognition of the succeeding transmit node.

Unit content $M_{\mathrm{s}}$ ought to be encoded using the node in our statement method. Meanwhile LCs recognizes conversion bases, node practices the conforming charge $b$ towards forming a novel communal basic $P_{\mathbb{1}}^{n}$ as shows in equation1:

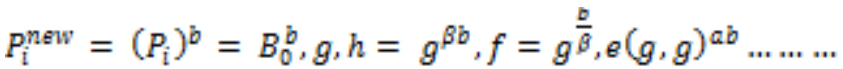

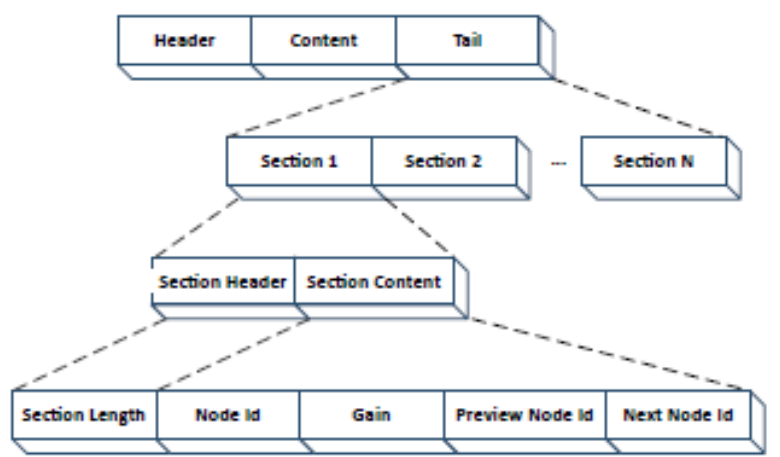

Fig. 5: The message structure.

Then, the encoded statement unit content may be attained using and represented in equation 2 :

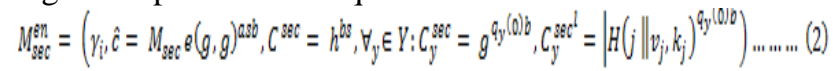

Whenever an LC obtains the statement, it fairly desires towards decrypting the statement unit by conversion basic $T_{i}$ as shows in equation 3 :

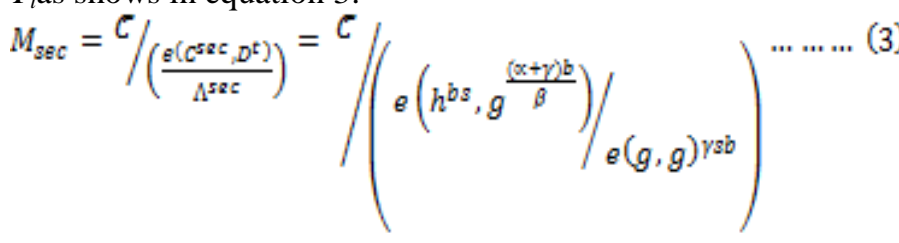

\section{SECURITY ANALYSIS}

In this segment, we investigate safety possessions of PCON.

\section{Privacy Preservation}

1) Data Privacy: We hire a characteristic-grounded cryptosystem towards encrypting messages afore they are referred out towards protecting information secrecy. Additional transmit nodes do not ensure the capability towards accessing messages in the structure. Aimed at LCs, conversion bases are well-defined. Grounded on them, LCs cannot infer the admission procedure as well as the isolated basic of some node. The purpose is that, a indiscriminate charge $b$ remains designated to describe the conversion basic grounded on a operator's communal basic, and value $b$ remains simply obtainable towards the operator possessing it. LCs might only interpret encoded basic $E_{i}^{\text {en }}$ using the conversion basic deprived of demanding extra data. Lacking $b$ as well as characteristic standards, LCs cannot infer the isolated basic though they scheme by mischievous nodes.

2) Attribute-based Privacy: For preserving characteristic-grounded secrecy, twofold approaches are employed: (a) we influence a safety grounded flexibility forecast procedure for protecting characteristic-grounded secrecy comprising connection interval approximation as well as self-esteem betweenness scheming. 
They might attain relationship consequences deprived of deliberating precise standards aimed at twofold nodes convoluted in the calculation procedure.

As they remain reassigned into indiscriminate standards for every transmission procedure, mischievous nodes remain incapable towards inferring those associated standards. (b) Individual characteristic-grounded data remains utilized to custom admission trees that ensure close relations with individual furtive bases. Deprived of clarifying them towards others informally, therefore, the operators maintain attention of their characteristic-grounded data.

3) Transaction Privacy: Towards ensuring enticement equality and safeguard operation discretion, we offer a new message configuration: (a) we practice statement units for recording node recompenses, as well as encode those units using the communal basic. Whereas additional nodes do not ensure the capability, LCs could decode statement units merely by means of the conversion basic. (b) We only practice LCs towards verifying information as well as assign recompenses aimed at customers that assure the equality of the structure.

\section{LATENCY ANALYSIS}

The latency in the system is the delay incurred in procuring the desired result. In this case it is caused by transmitting the data repeatedly. The process of reducing such delays is by carefully analyzing the data that is being transmitted. One such data that is retransmitted with every packet in this case is the key. One way to stop of the transmission to the key multiple times and yet maintain the security of the system is by adding Key Cache concept where key will be cache at node side to reduce transmission time of key exchange. The key can be cached by the receiver during the time the key remains the same. At the time of change in the key, the key can again be transmitted and the key cache process can be repeated.

Data key caching stores data keys and related cryptographic material in a cache. In the process of encryption or decryption of data, the receiver looks for a matching data key in the cache. If it finds a match, it uses the cached data key rather than generating a new one. Data key caching can improve performance, reduce cost, and help you stay within service limits as the application scales.

The application can benefit from data key caching if:

- It can reuse data keys.

- It generates numerous data keys.

- The cryptographic operations are unacceptably slow, expensive, limited, or resource-intensive.

\section{EXPERIMENTAL RESULTS}

Now-a-days COT is using to forward messages between two devices connected via internet. Devices will not have much storage space and heavy computation resources, all heavy computation and storage will happen at third party cloud server but this advantage will raise security issue as users data is processing or storing at third party server. To overcome from such issue, privacy preserving technique is used in which data is encrypted by using keys generated from users attributes such as username, age, gender etc. To decrypt that data user must have valid attributes and its not possible

for any malicious users to identify users private attributes. In opportunistic technique devices can communicate with cloud by using relay (neighbors) nodes, source will send data to neighbor node and whenever neighbor nodes comes in proximity of cloud server then data will be forwarded to cloud. If any malicious user receive data then he can't process that data without sender private attribute information.

With this technique selfish node (node which receive and drop data instead of sending to destination) problem also solved by implementing rewards concept. Source will choose relay node if it has high rewards point, to get points all nodes has to relay data to destination successfully. To become relay selfish node also has to send data to destination.

Two servers are being used in this context:

Remote Cloud: Responsible to store data and to process heavy computation task such as key generation, data receiving and decryption and verification.

Local Cloud: Responsible to receive data from all nodes which are available in its region and then transfer to remote cloud for processing.

Simulation: This application will perform simulation task of connecting to local cloud, remote cloud, message handling, data encryption and decryption etc. The below figure 6 shows and represents user interface.

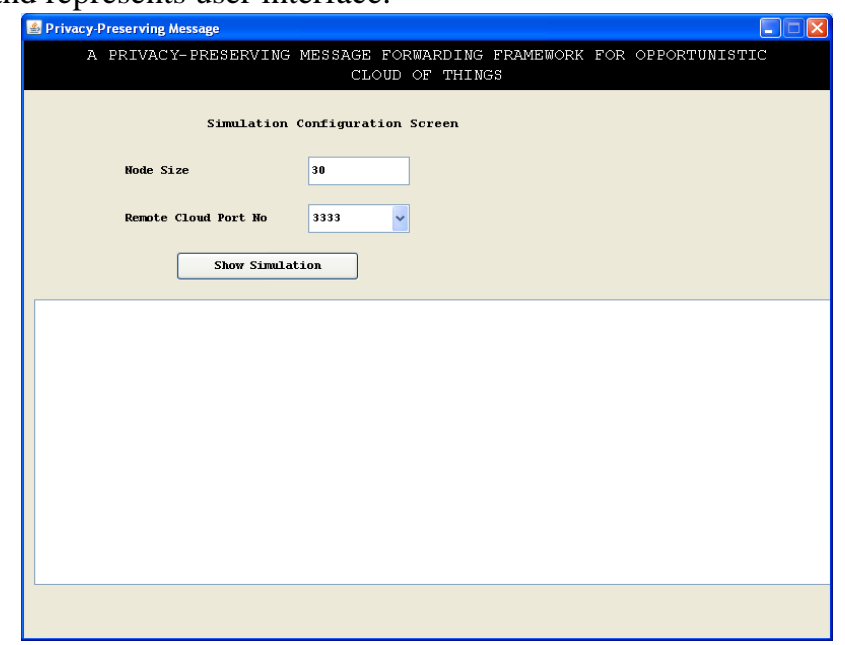

Figure6: Screenshot of the user interface created in JAVA. 30 users are created in random with random positions.

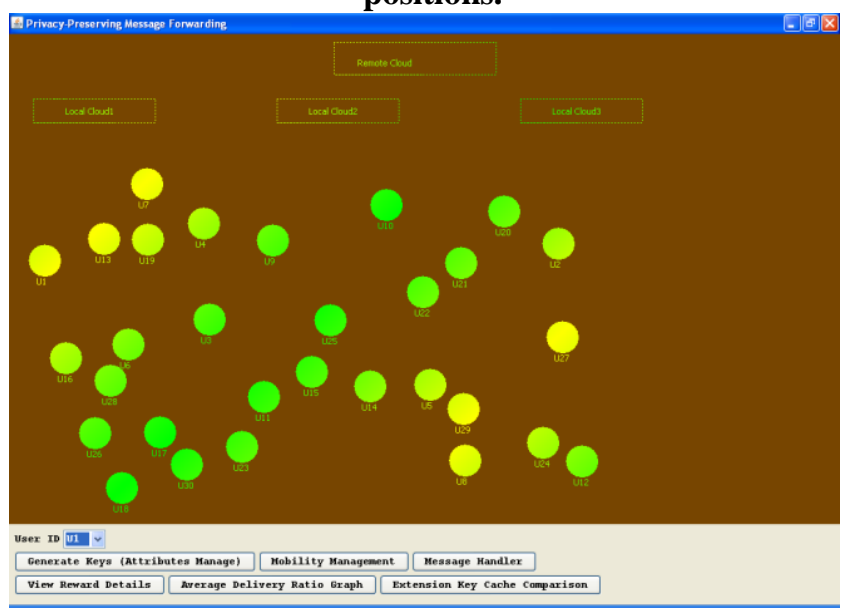

Figure7: User positions

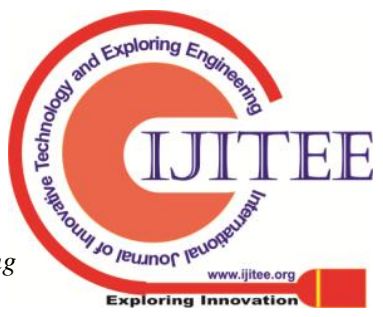


In above screen shows in figure 7, each circle represents as one user. 'Generate Keys' button will generate keys using user's attribute such as username. All users send data to relay node and relay node forward to local cloud and local cloud forward to remote cloud

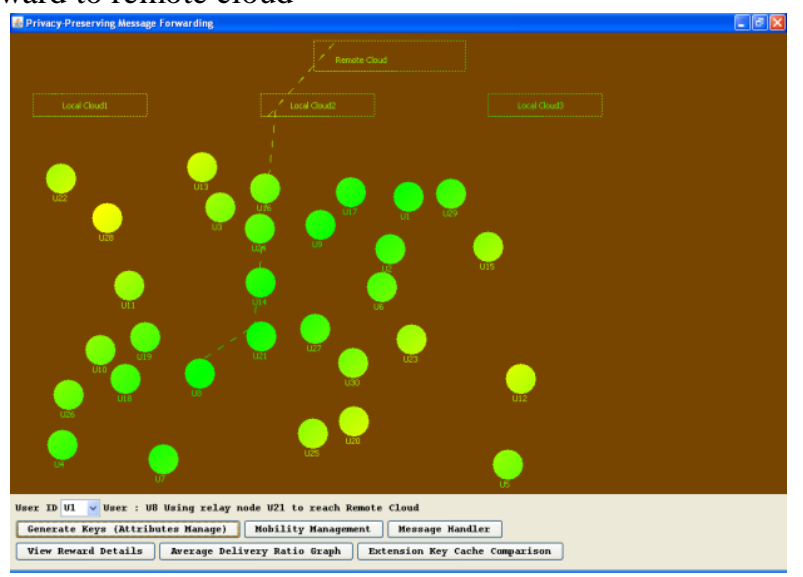

Figure8: Data communication process

In existing technique no security is there to identify malicious node and malicious node will drop data and decrease delivery ratio. In propose one based on valid keys only data will process and send and no drop will occur and shows in figure 8.

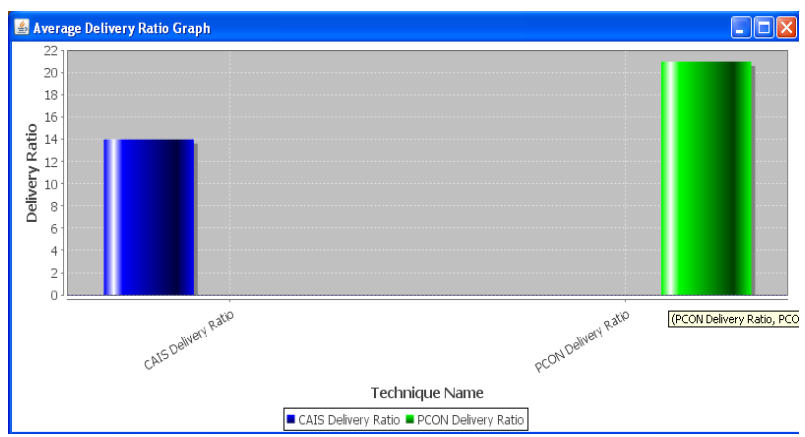

Figure9: Average delay ratio

Above screen shows in figure 9: $\mathrm{x}$-axis represents technique name PCON is Propose work and CAIS is existing work.

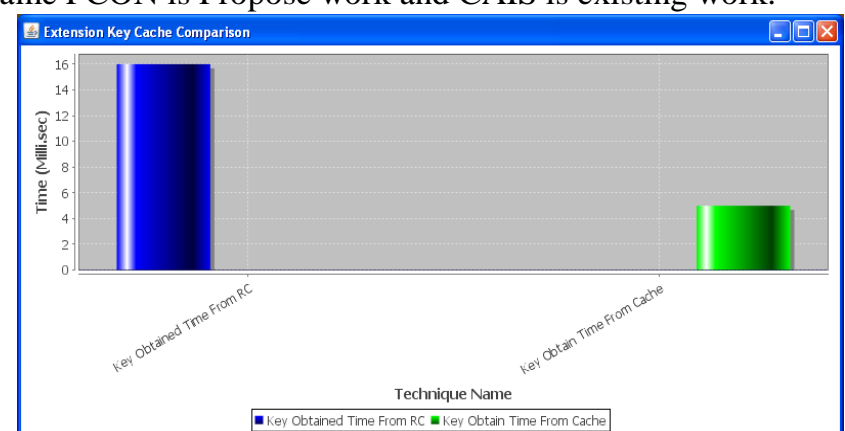

Figure10: Processing time for Key cache technique in comparison with the traditional technique and represents in figure 10. X-axis represents technique name and y-axis represents key obtain time.

\section{CONCLUSION}

In this paper, we propose a privacy-preserving message forwarding framework for opportunistic CoT with Key Cache concept is proposed. A two-layer cloud system, containing a remote and several local clouds, is developed to support secure message transmission. In order to defend against various attacks, both a security-based mobility prediction scheme and a cryptographic algorithm are investigated, not only specifying a fine-grained access control over messages, but also preserving individual privacy in message delivery. The delay caused due to re transmission of the key is reduced by the Key cache technique further improving the performance of the system.

\section{REFERENCES}

1. Z. Ning, F. Xia, X. Hu, Z. Chen, and M. S. Obaidat, "Social-oriented adaptive transmission in opportunistic Internet of smartphones," IEEE Transactions on Industrial Informatics, vol. 13, no. 2, pp. 810-820, 2017.

2. G. Fortino and P. Trunfio, Internet of things based on smart objects: Technology, middleware and applications. Springer, 2014.

3. Z. Ning, X. Hu, Z. Chen, M. Zhou, B. Hu, J. Cheng, and M. S Obaidat, "A cooperative quality-aware service access system for social Internet of vehicles," IEEE Internet of Things Journal, DOI: 10.1109/JIOT.2017.2764259, 2017.

4. G. Fortino, A. Guerrieri, W. Russo, and C. Savaglio, "Integration of agent-based and cloud computing for the smart objects-oriented IoT," in Proc. IEEE CSCWD, pp. 493-498, IEEE, 2014.

5. E. Toledano, D. Sawada, and A. Lippman, "COCAM: A collaborative content sharing framework based on opportunistic p2p networking," in Proc. IEEE CCNC, pp. 158-163, 2013.

6. Z. Ning, F. Xia, N. Ullah, X. Kong, and X. Hu, "Vehicular social networks: Enabling smart mobility," IEEE Communications Magazine, vol. 55, no. 5, pp. 16-55, 2017.

7. K. Ota, M. Dong, and S. Chang, "MMCD: cooperative downloading for highway vanets," IEEE Transactions on Emerging Topics in Computing, vol. 3, no. 1, pp. 34-43, 2015.

8. X. Wang, Z. Ning, and L. Wang, "Offloading in Internet of vehicles: A fog-enabled real-time traffic management system," IEEE Transactions on Industrial Informatics, Doi: 10.1109/TII.2018.2816590, 2018.

9. G. Aloi, G. Caliciuri, G. Fortino, R. Gravina, P. Pace, W. Russo, and C. Savaglio, "Enabling IoT interoperability through opportunistic smartphone-based mobile gateways," Journal of Network and Computer Applications, vol. 81, pp. 74-84, 2017.

10. W. Md, S. Mehdi, and G. Abdullah, "A survey on vehicular cloud computing," Journal of Network and Computer Applications, vol. 40, pp. 325-344, 2014.

11. Z. Ning, L. Liu, F. Xia, B. Jedari, I. Lee, and W. Zhang, "CAIS: A copy adjustable incentive scheme in community-based socially-aware networking," IEEE Transactions on Vehicular Technology, vol. 66, no. 4, pp. 3406-3419, 2017.

12. L. Yao, Y. Man, and Z. Huang, "Secure routing based on social similarity in opportunistic networks," IEEE Transactions on Wireless Communications, vol. 15, no. 1, pp. 594-605, 2016.

13. G. Fortino, W. Russo, C. Savaglio, M. Viroli, and M. Zhou, "Opportunistic cyberphysical services: A novel paradigm for the future Internet of things," in Proc. IEEE WF-IoT, pp. 488-492, 2018.

14. H. Zhu, M. Dong, and S. Chang, "ZOOM: scaling the mobility for fast opportunistic forwarding in vehicular networks," in Proc. IEEE INFOCOM, pp. 2832-2840, 2013.

15. Y. Li and S. Zhang, "Combo-Pre: A combination link prediction method in opportunistic networks," in Proc. IEEE ICCCN, pp. 1-6, 2015.

16. L. Zhang, B. Yu, and J. Pan, "GeoMob: A mobility-aware geocast scheme in metropolitans via taxicabs and buses," in Proc. IEEE INFOCOM, pp. 1279-1787, 2014.

17. H. Chen and W. Lou, "Making nodes cooperative: a secure incentive mechanism for message forwarding in DTNs," in Proc. IEEE ICCCN, pp. 1-7, 2013. 
18. T. Ning, Z. Yang, and H. Wu, "Self-interest-driven incentives for ad dissemination in autonomous mobile social networks," in Proc. IEEE INFOCOM, pp. 2310-2318, 2013.

19. Krifa, C. Barakat, and T. Spyropoulos, "MobiTrade: trading content in disruption tolerant networks," in ACM workshop on Challenged networks, pp. 31-36, 2011.

20. M. Xiao, J. Wu, and L. Huang, "Community-aware opportunistic routing in mobile social networks," IEEE Transactions on Computers, vol. 63, no. 7, pp. 1682-1695, 2014.

21. X. Fu, W. Li, G. Fortino, P. Pace, G. Aloi, and W. Russo, "A utilityoriented routing scheme for interest-driven community-based opportunistic networks," Journal of Universal Computer Science, vol. 20, no. 13, pp. 1829-1854, 2014.

22. J. Zhou, X. Dong, and Z. Cao, "Secure and privacy preserving protocol for cloud-based vehicular DTNs," IEEE Transactions on Information Forensics and Security, vol. 10, no. 6, pp. 1299-1314, 2015.

23. H. Li, H. Zhu, and S. Du, "Privacy leakage of location sharing in mobile social networks: Attacks and defense," IEEE Transactions on Dependable and Secure Computing, DOI:10.1109/TDSC.2016.2604383, 2016.

24. F. Li, H. Wang, and B. Niu, "A practical group matching scheme for privacy-aware users in mobile social networks," in Proc. IEEE WCNC, pp. 1-6, 2016.

25. M. Frustaci, P. Pace, G. Aloi, and G. Fortino, "Evaluating critical security issues of the IoT world: Present and future challenges," IEEE Internet of Things Journal, DOI: 10.1109/JIOT.2017.2767291, 2017.

26. X. Wang, L. Wang, and Z. Ning, "A privacy-reserved approach for message forwarding in opportunistic networks," in Proc. IEEE AINA, pp. 1070-1075, 2017.

27. P. Y. Zhang, Y. Kong, and M. C. Zhou, "A domain partition-based trust model for unreliable clouds," IEEE Transactions on Information Forensics and Security, vol. 13, no. 9, pp. 2167-2178, 2018.

28. R. Yu, J. Kang, X. Huang, S. Xie, Y. Zhang, and S. Gjessing, "MixGroup: Accumulative pseudonym exchanging for location privacy enhancement in vehicular social networks," IEEE Transactions on Dependable and Secure Computing, vol. 13, no. 1, pp. 93-105, 2016.

29. L. Guo, C. Zhang, and H. Yue, "A privacy-preserving social-assisted mobile content dissemination scheme in DTNs," in Proc. IEEE INFOCOM, pp. 2301-2309, 2013.

30. M. M. Prabhakaran and A. Sahai, Secure multi-party computation, vol. 10. IOS press, 2013.

31. W. Xiao, W. Bao, X. Zhu, W. Zhou, and P. Lu, "Improving the performance of data sharing in dynamic peer-to-peer mobile cloud," in IEEE International Conference on Parallel and Distributed Systems, pp. 743-752, 2016.

32. Z. Ning, X. Wang, X. Kong, and W. Hou, "A social-aware group formation framework for information diffusion in narrowband Internet of things," IEEE Internet of Things Journal, vol. 5, no. 3, pp. 1527-1538, 2017.

33. D. M. Nessett, "Offloading cryptographic processing from an access point to an access point server using otway-rees key distribution," July 4 2006. US Patent 7,073,066.

34. C. Yao, "Protocols for secure computations," in Proc. IEEE SFCS, pp. 160-164, 1982.

35. Q. Xu, Z. Su, and S. Guo, "A game theoretical incentive scheme for relay selection services in mobile social networks," IEEE Transactions on Vehicular Technology, vol. 65, no. 8, pp. 6692-6702, 2016.

36. A.-K. Pietilainen, E. Oliver, and J. LeBrun, "MobiClique: Middleware" for mobile social networking," in ACM Workshop on Online Social Networks, pp. 49-54, 2009. 